\title{
The Next Era: Flourish of National Health Care \& Medicine with the World Leading Artificial Intelligence
}

\author{
Jian $\mathrm{Wu}^{*}$ \\ College of Computer Science and Technology, Zhejiang \\ University, Hangzhou 310027, China
}

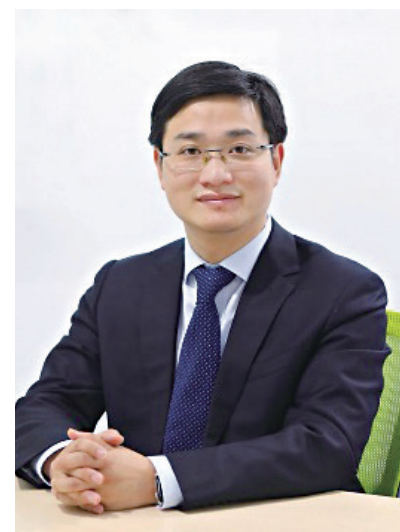

Prof. Jian Wu graduated from Zhejiang University (ZJU) and obtained his Ph.D. degree at the College of Computer Science and Technology, ZJU. He is currently the vice president of National Academy of Health and Medical Big Data at ZJU, the director of the Real-doctor Artificial Intelligence (AI) Research Center of ZJU, and is also serving as members of China Computer Federation (CCF)'s Committee for Elite Young Professionals, and the Ministry of science and technology's key field innovation team. Prof. Wu's research focus on AI in Medicine. In recent years, he has presided one National Science and Technology Support project, five China's National Natural Science Foundation projects, and three National High Technology Research and Development Program (863 program) projects. He has received 32 licensed patents and published over $100 \mathrm{SCI} / \mathrm{EI}$ referenced papers in this field. His received honors and awards include the 100 Most Influential Domestic Academic Paper Award in 2008 and 2009, the CCF Excellent Paper Award in 2017, the First Prize of Science Technology Progress Award of Ministry of Education in 2007, the Second Prize of National Science Technology Progress Award in 2010, the First Prizes of Science Technology Progress Award of Zhejiang province in 2008 and 2014, and the Supreme prize of China General Chamber of Commerce's Science and Technology Award.

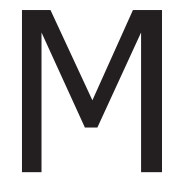

ODERN medical diagnosis and practice heavily rely on biological data and information from patients' body. The progress of biomedical sensor, material and mathematical technology provided ever-increasing methods to gather data. While providing more choices and more comprehensive picture of patients' conditions to doctors and practitioners, these progresses also require more labor efforts to read, analyze, and make decisions based on those data. It is very difficult for the medical human resources to grow at a speed that matches such need for diagnosis-related expert knowledge. The shortage of expertise has caused long wait-

*Correspondence: wujian2000@zju.edu.cn ing time for check report and fatal misjudged diagnosis in public health system, and it will compromise our ability to move towards a more precise, more personalized and more efficient future of medicine.

The most helpful solution at present time is the introduction of artificial intelligence (AI) into medicine. AI has experienced rapid development in recent years due to availability of big data and deep learning. ${ }^{1}$ It has revolutionized computer vision, natural language processing, and goal-directed task solving. ${ }^{2}$ There have been a lot of AI applications in medical practices and health care, ${ }^{3,4}$ including image proof reading, ${ }^{5}$ medical records structuring, ${ }^{6}$ risk prediction and management, ${ }^{7}$ etc. AI not only can provide consistent and stable diagnosis/analysis ability that 
can be easily extended, but is also useful in training of medical skills, thus help solving the shortage issue from human resource side too.

This special issue focuses on recent advances of medical AIs. It includes review articles for the key AI algorithms and technologies used in medicine, the current status of computer-aided diagnosis in several fields or diseases which are heavily studied, the examples of applications of AI in various forms, and the potential way that AI can cooperate and change the common practice of health system. We hope this issue can give experts and practitioners in medicine a comprehensive view of the status quo of medical AI and inspire more ideas for further development of this realm.

\section{REFERENCE}

1. LeCun Y, Bengio $Y$, Hinton G. Deep learning. Nature 2015 ; 28;521(7553):436-44. doi: 10.1038/nature14539.

2. Mnih V, Kavukcuoglu K, Silver D, et al. Playing atari with deep reinforcement learning. https://arxiv.org/ abs/1312.5602. Published December 19, 2013; accessed June 9, 2019.

3. Esteva A, Robicquet A, Ramsundar $B$, et al. A guide to deep learning in healthcare. Nature Med 2019; 25(1):24-9. doi: 10.1038/s41591-018-0316-z.

4. He J, Baxter SL, Xu J, et al. The practical implementation of artificial intelligence technologies in medicine. Nature Med 2019; 25(1):30-6. doi: 10.1038/s41591018-0307-0.

5. Greenspan H, Ginneken BV, Summers RM. Deep learning in medical imaging: overview and future promise of an exciting new technique. IEEE Trans Med Imaging 2016; 35(5):1153-9. doi: 10.1109/TMI2016.2553401.

6. Ravi D, Wong C, Deligianni F, et al. Deep learning for health informatics. IEEE J Biomed Health Informatics 2016; 21(1):4-21. doi: 10.1109/JBHI.2016.2636665.

7. Ravizza S, Huschto T, Adamov A, et al. Predicting the early risk of chronic kidney disease in patients with diabetes using real-world data. Nature Med 2019; 25(1):57-9. doi: 10.1038/s41591-018-0239-8. 